\title{
QUANTIFICATION AND EVALUATION OF HUMAN TACTILE SENSE TOWARDS FABRICS
}

\author{
N. PAN \\ Fiber and Polymer Science, Biological and Agricultural Engineering Department, University of California, Davis, CA, USA \\ ABSTRACT \\ This paper introduces a novel approach for evaluating fabric sensory responses. First, the problems in existing \\ techniques are analyzed, and by comparing with successful cases in assessing human feeling towards cold/warmth \\ and human color sense, a more scientific scheme based on the computer pattern recognition technique is exhibited \\ and the details of the measurements, data processing and calculation of the ultimate parameters are introduced. \\ The actual prototype of the instrument and related details are also provided. Finally, it is shown that the technique \\ can also be utilized to evaluate some visual attributes such as drape and wrinkle recovery. \\ Keywords: fabric hand, feature extraction, human tactile sense, measurement and evaluation.
}

\section{TACTILE SENSORY EVALUATION OF FABRICS}

Tactile sense or the sense of touch has been an important aspect of the human interactions with the environment. The study of tactile sense, or the haptics, has received tremendous attentions for its potential applications in computer virtual reality, remote surgery and manipulation as well as in packaging techniques, sports products design and automobile industries $[1,2,3,4,5,6,7,8,9,10]$. It also concerns other specialized areas such as risk evaluation, investment evaluation and safety $[11,12]$.

Perhaps the first sensory evaluation applied to product quality assessment is on food, clothing and cosmetic products $[13,14,15,16,17]$. Fabric hand, or handle as it is often called, is defined as the human tactile sensory response towards fabric, which involves not only physical but also physiological, perceptional and social factors; this very fact complicates the process of fabric hand evaluation tremendously $[18,19,20,21,22]$.

The importance of this fabric quality perceived through tactile sense is indisputable. It is hard to imagine a consumer buying a textile product without touching it, and a poor hand is often the reason why a consumer rejects a product; one well-known example is polyester fiber; it acquired such a bad image during its early stages mainly because of its poor fabric hand. The success of any new fiber, new finish or new textile product is largely dependent on the acceptance of its fabric hand. However, assessment of this quality attribute until now still largely relies on human tactile sensory judgment, which in many cases is not reliable. Furthermore, while it is common knowledge to textile scientists that the physical aspect of the fabric hand is attributed to the properties of the fabric, there is no approach by which this aspect can be measured directly. This is mainly due to the fact that even the physical fabric hand is basically a reflection of the overall fabric quality, attributed to many individual fabric properties.

The first attempt to study the phenomenon of fabric hand was initiated by Binns in 1926 [18], by employing people with a wide range of backgrounds in order to investigate the hand characteristics among different groups and individuals. Because of the importance of fabric hand, there have been at least four well-attended international conferences (in the year 1981 in Japan, 1983 in Australia, 1985 in Japan and 1988 in Hong Kong) exclusively devoted to this subject, pushing forward significantly the research in this area.

The direct sensory evaluation method for assessing fabric hand is probably the most logical. As Brand [20] stated, 'The aesthetic concepts [of fabrics] are basically people's preferences and should 
be evaluated subjectively by people.' However, this apparent common-sense approach immediately runs into difficulties such as finding the most appropriate judges: experts or untrained consumers? There is difficulty with the communication between judges, the low assessment sensitivity, the adverse effects due to personal subjectivity and preference, and the scaling shift associated with the fatigue and conditioning of the human senses. The conclusion has been that a reliable sensory evaluation of fabric hand is possible, but obviously the method does not facilitate rapid development of textile products. Thus, an instrumental approach becomes the next logical choice.

Peirce in 1930 [19] first proposed to evaluate fabric hand based on physical measurement data. Since then, there have been several attempts to use instruments to measure fabric hand. All these efforts climaxed in 1970 when Kawabata and his co-workers in Japan developed a KES-FB system [23, 24] for fabric hand evaluation. This entire system is composed of four instruments, each measuring a few different fabric properties such as tensile and shearing, bending, compression, and surface properties at low stress, simulating the forces encountered when handling a fabric. The fundamental principle of this system is to connect the measured 16 mechanical properties of a fabric directly to its Japanese hand preference through multivariate statistical regression analysis. However, the system is expensive and is beyond the reach of most small companies/users; also the time and effort required to complete the measurement of all 16 mechanical properties ( 9 of them involve both warp and weft directions, doubling the number of measurements) are in many cases unacceptable. Furthermore, because of the subjectivity of human preference, this system failed to offer an adequate solution for fabric hand assessment in countries other than Japan, and there are still many other problems associated with this system as described in the papers $[25,26,27]$.

In 1990, several scientists in Australia built another instrument system called the FAST system [28], which is basically a simplified version of the Japanese KES-FB system and therefore has the similar problems. Besides, both systems are time consuming.

More important, the results from these systems are not accurate reflections directly or indirectly of the human tactile response towards fabrics.

Successful resolution of the fabric hand evaluation problem will not only provide a powerful quality assurance method for the textile industry, it will also offer solutions to other consumer product questions, where product quality relies on tactile sensory evaluation, e.g. softness of pillows and comfort of pants. In addition, it has some implications or may shed light on our understanding of the relationship between physical stimuli and physiological, psychological and perceptual response.

\section{SUCCESSFUL EXAMPLES}

Before proceeding further, let us look at a few seemingly successful examples that should be helpful in our attempts to measure and evaluate human sensory responses. First, for our sense of cold and warmth, we rely on an artificially defined physical quantity termed temperature. Thus, by measuring the ambient temperature $T^{0}$ at a given level of relative humidity RH and air flow speed $V$, we can assess or estimate our own sense of coldness accurately to the degree of satisfaction for most practical purposes when stepping into that environment, although the sense of coldness, like all other sensory responses, is highly personal and subjective.

Another example is our sense of color (chromaticity). It is now widely accepted that a single set of three appropriately chosen primary colors is sufficient to match the color appearance of any stimulus for a trichromat. Normal human color vision is trichromatic, but some other organisms, e.g. pigeons, have tetrachromatic color vision: they require sets of four primary colors to produce complete color matches. Other organisms and some color deficient human beings are dichromats: they require only sets of two primary colors to produce complete color matches. The human visual sense of color is obviously a highly subjective matter, just like the human tactile sense. However, the modern 


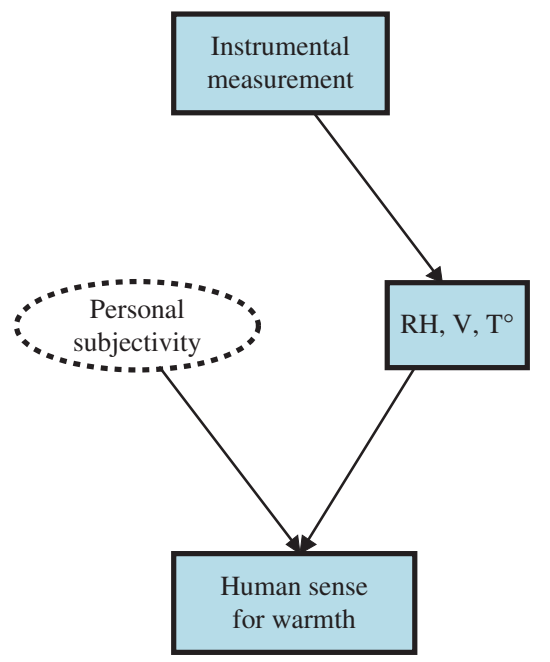

(a)

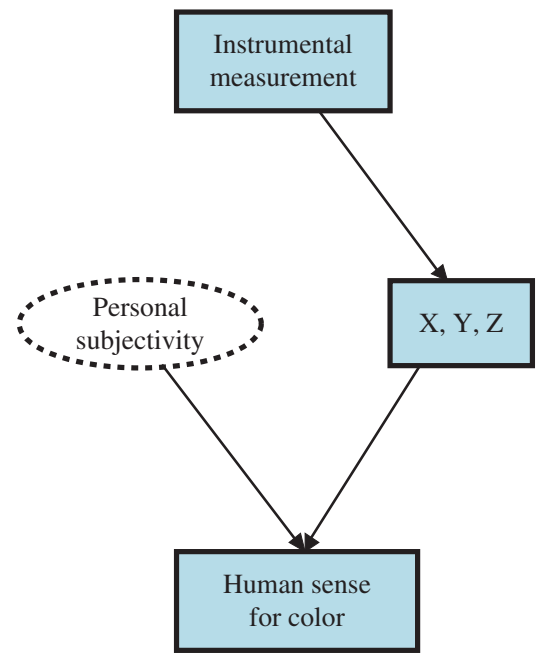

(b)

Figure 1: Successful attempts: (a) human warmth/cold and (b) color evaluation.

chromatology has to a great degree overcome this problem of color sense evaluation by defining a particular color as a mixture of three primary values $X, Y, Z$ of given spectrums.

Figure 1(a) and (b) may reveal the secret of the above successful cases on how to scientifically deal with human subjectivity. Any natural phenomenon cannot be studied scientifically until it can be measured instrumentally. However, there is no well-established instrumental technology by which human sensations can be measured quantitatively. The major obstacle is the embedded personal subjectivity, which is the implacable foe for any attempt of direct instrumental measurement and quantification. From Fig. 1, we learn that the secret for success is to separate the human subjectivity and the physical quantities that evoke the human sensation, yet are stable, clearly defined and measurable instrumentally. So the key to evaluate the human tactile sense seems to be the identification, isolation and measurement of the physical entities that stimulate the sensation. Thus, if we examine all the previous attempts to measure the fabric hand instrumentally, the common blunder seems to be that all these attempts try to use instruments directly to measure something which is a mixture of some physical attributes combined with personal judgment and preference, and therefore subject to change. Hence, this approach is self-contradictory and doomed to fail.

Furthermore, although it is common knowledge to textile scientists that fabric hand is attributed to a fabric's physical properties, there is no approach by which it can be measured directly. This is mainly due to the fact that fabric hand is basically a reflection of the overall fabric quality, consisting of many individual fabric properties.

\section{ISSUES TO BE ADDRESSED}

As demonstrated in earlier work [26, 27], the nature of fabric hand can be described by two seemingly contradictory aspects: on one hand, it represents an esthetic concept, a reflection of people's sensory preferences; on the other hand, it is indeed a subjective attribute depending on market background, consumer perception and product types. Despite this seemingly intrinsic barrier, the assessment of fabric hand is still meaningful, partly because it is extremely desirable in many practical cases. Postle and others have conducted a series of projects to examine and demonstrate this essential nature of 
fabric tactile response $[29,30,31]$ among people from several different countries. By recruiting eight expert judges each from five countries including Australia, India, Japan, New Zealand and USA in addition to eight ordinary consumers from India, where the final survey was conducted, and collecting a large number of fabric samples for both winter suits and summer dress, the researchers asked the panels to rank and score the fabrics based on the hand preferences. To show the level of variation and consistency between judges within and between each of the countries surveyed, the mean correlation coefficients of the fabric ranking scores in both cases were computed $[29,30]$ as shown in Table 1.

The results in Table 1 reveal that for the same fabrics, acceptability varies with judges from different countries. Especially for summer fabrics, the Japanese panel of judges showed a marked disagreement or even opposite preferences, indicated by the negative correlations. This kind of diversity is a psychological fact, and until psychologists can derive a rigorous formulation for the fundamental relationship between physical stimuli and the human sense of perception, it will be impossible for physical scientists to solve the problem of evaluating fabric hand completely using a purely analytical method.

However, the high values of the within-group correlation coefficients suggest that these expert judges definitely agree, within each national group, about the hand rating of those fabrics; even the untrained consumer judges show reasonable agreement between themselves. It is just this fact of the local reliability or the consistency of fabric hand preference that provides the meaning and possibility of fabric hand evaluation.

Finally, it is highly intuitive to think that the thermal properties of the fibers would play a critical role since the sensation of warmth of a fabric is part of the tactile responses. In fact, the tactile sense is a reflection of contact transient, and is hence highly related to the so-called effusivity $E=\sqrt{k \rho c_{p}}$ of the material, where $k$ is the thermal conductivity $(\mathrm{W} / \mathrm{m} \mathrm{K}), \rho$ is the density $\left(\mathrm{kg} / \mathrm{m}^{3}\right)$ and $c_{p}$ is the specific heat capacity $(\mathrm{J} / \mathrm{kg} \mathrm{K})$ of the material. A surface with a higher effusivity value feels cooler. Obviously, the narrow range of the thermal conductivities $k$ of various textile fibers $(0.1-0.3 \mathrm{~W} / \mathrm{m} \mathrm{K})$ cannot account for the vast scope of the cooling sensation received by touching different fabrics. However, since the material density $\rho$ and the specific heat capacity $c_{p}$ are either determined by or heavily dependent on the structure of the fabric, the latter plays a much dominant role here. This explains why fabrics made of the same fiber often exhibit entirely different skin contact sensations. For instance, a winter coat and a summer T-shirt can be made from wool fibers of similar thermal conductivities.

This to a large degree validates the hypothesis that fabric tactile sense is a result of various structural, mechanical and surface parameters [32,33], which dictate the fabric warmth sense, and the effect of fiber thermal conductivity is negligible.

Table 1: Correlation coefficients between international panels of fabric ranking scores [29, 30].

$\begin{array}{lccccc} & \text { Japan } & \text { Australia } & \text { New Zealand } & \text { India (winter) } & \text { USA } \\ \text { Japan } & & & & & \\ \text { Australia } & -0.34 & 0.85 & 0.76 & 0.82 & 0.80 \\ \text { New Zealand } & -0.30 & 0.82 & 0.86 & 0.91 & 0.87 \\ \text { India (summer) } & -0.41 & 0.78 & 0.76 & 0.83 & 0.83 \\ \text { USA } & -0.33 & 0.81 & 0.74 & 0.76 \ldots \ldots\end{array}$

Note: Table 1 is a combination of two tables. Since country A vs. country B is the same as country B vs. A, i.e. the tables are symmetrical, we only need one half of the table for each case (summer and winter fabrics, respectively). Therefore, the dotted line divides the table into two halves, emphasizing that the top and bottom halves are not the same data sets. 
Thus, for any attempt of instrumental evaluation of the tactile sense, the following issues have to be addressed [32, 33]:

1. what is to be measured for necessary and sufficient information?

2. how to make the measurement at low cost with quick and easy operation?

3. interpretation of the data including establishment of some objective indices that have clear physical meanings to the users on one hand and show connection to the sensory preference on the other.

\section{THE METHODOLOGY}

\subsection{Measurement scheme-the fabric exaction method}

Looking at the way fabrics are handled by consumers before they make a purchase decision, the fabric is deformed at various stress states so as to generate a tactile sensation in the fingers. It was thus recommended by Peirce first [19] and then Kawabata [23] and Postle [32] that the following characteristics of fabric deformation have to be captured for any measurement attempt:

1. low yet complex stresses at large deformation;

2. nonlinearity;

3. friction/hysteresis.

Two classes of measurement approaches have been adopted by existing methods and systems. The first approach is to test a fabric sample as a whole only once; tests in this category include the cantilever method, the heart-loop method, etc., both described in ASTM D 1388, and the circular bend test in ASTM D 4032. The major problem with tests of this type is that they provide only a single parameter that cannot completely define a phenomenon as complex as fabric hand. Moreover, none of the parameters targeted can serve as a consistent indicator to reflect human tactile sensation. The second group consists of both the KES [24] and the FAST [28] systems which, although capable of measuring various mechanical and structural properties of a fabric sample, still fail to associate their measurements with human sensory responses effectively.

The testing method used per se in this paper is not a new idea at all; it has been a common practice for many years by ladies in certain parts of the world when searching for a desired scarf at a market. They would take off their rings and pull out a scarf through the ring, judging the overall quality of the scarf based on the resistance during the pulling out process. This technique, as illustrated in Fig. 2, is nowadays called the fabric extraction technique [34, 35, 36].
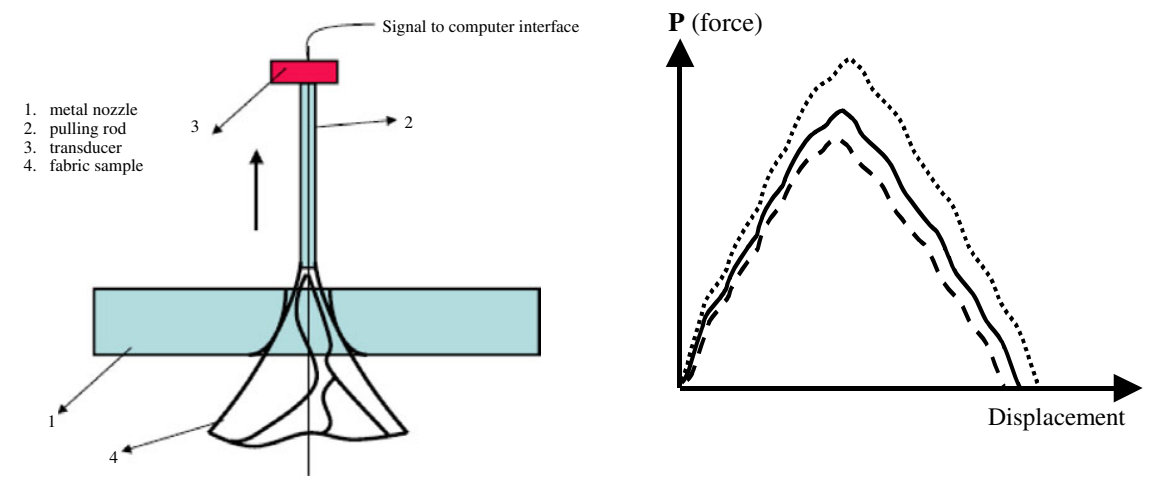

Figure 2: The fabric extraction technique and force-displacement curves for three different fabrics. 
For a properly designed nozzle, if we examine the fabric extraction process carefully, we will find that during the process the sample is deformed under a very complex yet low stress state including tensile, shearing and bending as well as frictional actions, similar to the stress state when we handle a fabric. Consequently, all the information related to fabric hand is reflected by the resulting loaddisplacement extraction curve. Previously, researchers made use of only one feature of the curve, e.g. the peak or the slope at a point [34], and discarded the rest of the information. If we can identify and derive all this information and classify it in terms of known fabric attributes, the significance is indisputable, and this is exactly what we have attempted to do [36, 37].

\subsection{The pattern recognition method}

Once the fabric extraction process is done and an extraction curve similar to the one shown in Fig. 2 is obtained, several following tasks need to be accomplished [36, 37].

4.2.1 To discover or reduce the dimensionality of the data set

The fact that this is a continuous curve presents a problem in that all the points on the curve are more or less correlated, as we can predict where a given data point will be located by knowing the locations of its prior and/or subsequent points; the closer the neighboring points, the better the prediction or the higher the correlations between them. These ominously existing correlations among the data imply a redundancy of information contained in the data points. Therefore, for both simplicity and facilitation of further analysis, we have to eliminate the redundancy and consequently reduce the number, or the dimensionality, of the data set.

4.2.2 To identify new meaningful underlying variables

With fewer but condensed data points (or features, as they are usually called) retained, we need to determine their identity or physical meanings so as to interpret the results. Thus, for different fabrics, there are different extraction curves, and detection of the differences, or determination of the physical significance of the features, are just what the pattern recognition techniques deal with [38, 39].

According to the Karhunen-Loeve (K-L) orthonormal expansion theorem [38] of the statistical pattern recognition technique, a random vector $X=\left(X_{1}, X_{2}, \ldots, X_{n}\right)$, with mean vector $E(X)=E$ and covariance matrix $\operatorname{Var}(X)=V$, can be replaced by an orthogonal vector $Y$ through a matrix transformation

$$
Y=X R
$$

with an acceptable information loss $\leq e_{m}$, where

$$
Y=\left(Y_{1}, Y_{2}, \ldots, Y_{p}\right)
$$

and

$$
R=\left(R_{1}, R_{2}, \ldots, R_{p}\right)
$$

$R_{1}, R_{2}, \ldots, R_{p}$ are the $p$ eigenvectors corresponding to the $p$ prior eigenvalues of the descending sequence $\lambda_{1}>\lambda_{2}>\ldots>\lambda_{p}$ of the covariance matrix $V$ of $X$. The dimension $p$ of both matrices $Y$ and $R$ is determined by [39]

$$
p=\frac{\sum_{i=1}^{p} \lambda_{i}}{\sum_{k=1}^{n} \lambda_{k}} \leq e_{m},
$$

where $\operatorname{Tr} V=\sum_{k=1}^{n} \lambda_{k}$ is the trace of the covariance matrix $V$. 
The new vector $Y$, called the feature vector of the original vector $X$, has the following interesting properties: (i) in general, the dimensions of $Y$ are much smaller than those of $X$, i.e. $p<n$; (ii) The components of $Y$ are all uncorrelated with each other, i.e. $Y$ is orthogonal; (c) the relative importance $W_{i}$ of each component $Y_{i}(i=1,2, \ldots, p)$ in expressing the original vector $X$ is represented by the corresponding eigenvalue $\lambda_{i}$ associated with the eigenvector $R_{i}$ in terms of the ratio

$$
W_{i}=\frac{\lambda_{i}}{\sum_{k=1}^{p} \lambda_{k}}
$$

\subsection{Final parameters and their physical meanings}

By testing fabrics using the extraction method, the extraction curve (displacement vs. extraction force) is discretized into a data set $X$ which is then transformed into the feature vector $Y$. The dimension change and the resulting error for a specific case are listed in Table 2, and the details of the eigenvalues, the individual weight for each corresponding feature component $Y_{i}$ and the total contributions accounted by $Y$ are provided in Table 3 .

We have tested fabrics of extensive weight ranges and different types, and concluded that $p=8$ is more or less acceptable in every case. However, the relative weight for each feature varies depending on the fabric types, and should be determined and saved for further use.

The power of the $\mathrm{K}-\mathrm{L}$ transformation is clearly shown; by reducing the original data dimension from 110 to 8 , only $11 \%$ of information is lost. Furthermore, no correlations exist among the 8 components, i.e. the human tactile sensory response space has 8 dimensions or 8 independent attributes. The next tempting and logical step is to determine the physical meanings of these attributes, if any. Since there is no theoretical means to complete this task, we have to turn to experimental approaches. For the same fabrics used in Tables 2 and 3, we obtained the feature set $Y_{i}(i=1,2, \ldots, 8)$. Next, because of the lack of better standards, we calculated the corresponding set of the so-called three primary hand values including stiffness, smoothness and softness for the same fabrics based on Kawabata's method [24]. Using correlation analysis, we found that the first three components of the $Y$ set are highly correlated with the primary hand values [37] and in that the same order as well, as shown in Table 4.

This result was then compared many times with the tactile sensory approach using fabrics with distinctly different fabric features; for instance, two fabrics with extremely different stiffness to validate if the calculated stiffness $Y_{1}$ values indeed indicate a consistent trend in fabric stiffness change.

Table 2: Dimension change and the resulting error.

\begin{tabular}{lc}
\hline Dimension of $X$ & $n=110$ \\
Dimension of $Y$ & $p=8$ \\
Allowable error [39] & $e_{m}=11 \%$ \\
\hline
\end{tabular}

Table 3: The ranked eigenvalues, the individual weights and the total contributions accounted.

\begin{tabular}{lcccccccc}
\hline & 1 & 2 & 3 & 4 & 5 & 6 & 7 & 8 \\
\hline$\lambda_{i}$ & 4.032 & 3.120 & 2.304 & 1.440 & 1.200 & 0.816 & 0.672 & 0.656 \\
$W_{i}$ & 0.252 & 0.195 & 0.144 & 0.090 & 0.075 & 0.051 & 0.042 & 0.041 \\
$\sum W_{i}$ & 0.252 & 0.447 & 0.591 & 0.681 & 0.756 & 0.807 & 0.849 & 0.890 \\
\hline
\end{tabular}


Table 4: The first three feature components and their physical meanings.*

\begin{tabular}{lcl}
\hline Component feature & Associated weight & Primary hand \\
\hline$Y_{1}$ & $W_{1}=0.252$ & Stiffness \\
$Y_{2}$ & $W_{2}=0.195$ & Smoothness \\
$Y_{3}$ & $W_{3}=0.144$ & Softness \\
\hline
\end{tabular}

*Note that these features are mathematically determined but the fabric hand attributes such as stiffness and softness are from human sensory responses that are inevitably intertwined with each other. It is therefore illogical to attempt associating these features with the hand attributes, no less than associating individual primary colors with the hue or tune of the resulting color perception. However, to make the current system easier to adopt, we have spent a huge amount of time to establish some connection between two groups that define the same fabric hand albeit through different paths. After all, these terms (and their equivalences in other languages) have been used to describe fabric hand for several hundreds or even thousands of years.

Meanwhile, we also adopted other physical means to verify the results. For instance, by altering the exposure time of a fabric to plasma treatment, we can change the fabric surface friction in a monotonic direction without affecting the other properties too much. Thus, we can use these treated fabrics to calibrate the smoothness value $Y_{2}$. With ever advancing techniques, we are confident that we can conduct such checking and calibration more effectively for vastly diverse fabric types.

To date, we have defined only the first three features of the set $Y_{i}(i=1,2, \ldots, 8)$ shown in Table 4. The remaining five are only included in calculating an overall value described below, until such practical needs for more well-defined fabric hand features arise.

\subsection{An overall value}

Besides the three fabric hand descriptors defined in Table 4, there is an obvious need for a single index to represent or describe the fabric hand, even though the phenomenon is a complex and multifaceted one. First, the procedure of tactile sensory assessment itself is in essence a subconscious transformation from the various physical stimuli to a single one-dimensional assessment. Moreover, for practical application, such a comprehensive index often suffices for quality communication.

When comparing differences between individual subjects, the concept of distance is often a useful one. Based on the feature set $Y$ for each fabric sample, we can readily calculate a Euclidean distance between two samples, a greater distance indicating a larger overall difference in terms of the feature set of fabric properties. Furthermore, if a sample $k$ is chosen as a standard reference, the distances of other fabrics to $k$ then become comparable and can be used as an objective measure of the fabric hand.

However, although convenient, the distance value thus calculated has a serious flaw; it is highly possible that fabrics with identical values of distance as defined above possess different characteristics of total hand. To alleviate this fault, it is necessary to determine the weight of each feature component and define a weighted distance.

Let us consider the standard fabric $k$ with a known feature set $Y_{k}$

$$
Y_{k}=\left(Y_{k 1}, Y_{k 2}, \ldots, Y_{k p}\right) \text {. }
$$

Then, for two other fabrics to be evaluated

$$
Y_{1}=\left(Y_{11}, Y_{12}, \ldots, Y_{1 p}\right) \text { and } Y_{2}=\left(Y_{21}, Y_{22}, \ldots, Y_{2 p}\right) \text {, }
$$


the weighted Euclidean distance [25] can be calculated for both fabrics, with fabric $k$ as the reference, by considering all $p=8$ feature components as

$$
\operatorname{PH}(i)=\sqrt{\sum_{j=1}^{p} w_{j}\left(Y_{i j}-Y_{k j}\right)},
$$

where $i=1$ and 2 corresponding to fabrics 1 and 2 to be evaluated.

Two hand value systems can thus be established based on how the reference fabric $k$ is chosen.

1. Using a system-designated reference fabric: The PH value thus calculated is a purely objective index that only specifies the hand difference. A smaller PH value represents a quality closer to the reference fabric.

2. Using customer-provided preferred fabric: The PH value now becomes an indicator of hand preference and provides hand preference ranking for the customers. The diversions among consumer groups in terms of fabric hand preference can hence be dealt with by allowing the consumers to inform the instrument system of their preferences. In fact, even for the tactile sensory assessment method, it presumes the existence of a preference standard in the judge's conscious or subconscious mind. Indeed, in the absence of such an existing preference, the whole concept of handle evaluation becomes meaningless.

Furthermore, we can use a graphic technique proposed in [40] to fingerprint all the fabrics in comparison with the reference fabric as shown in Fig. 3, in which the coordinate system consists of four axes representing the three fabric hand attributes and the PH value and Sample 3 is taken as the reference. A fingerprint is unique to a fabric just like a human fingerprint is to a person. More convenient analyses can be made and more specific information can be derived.

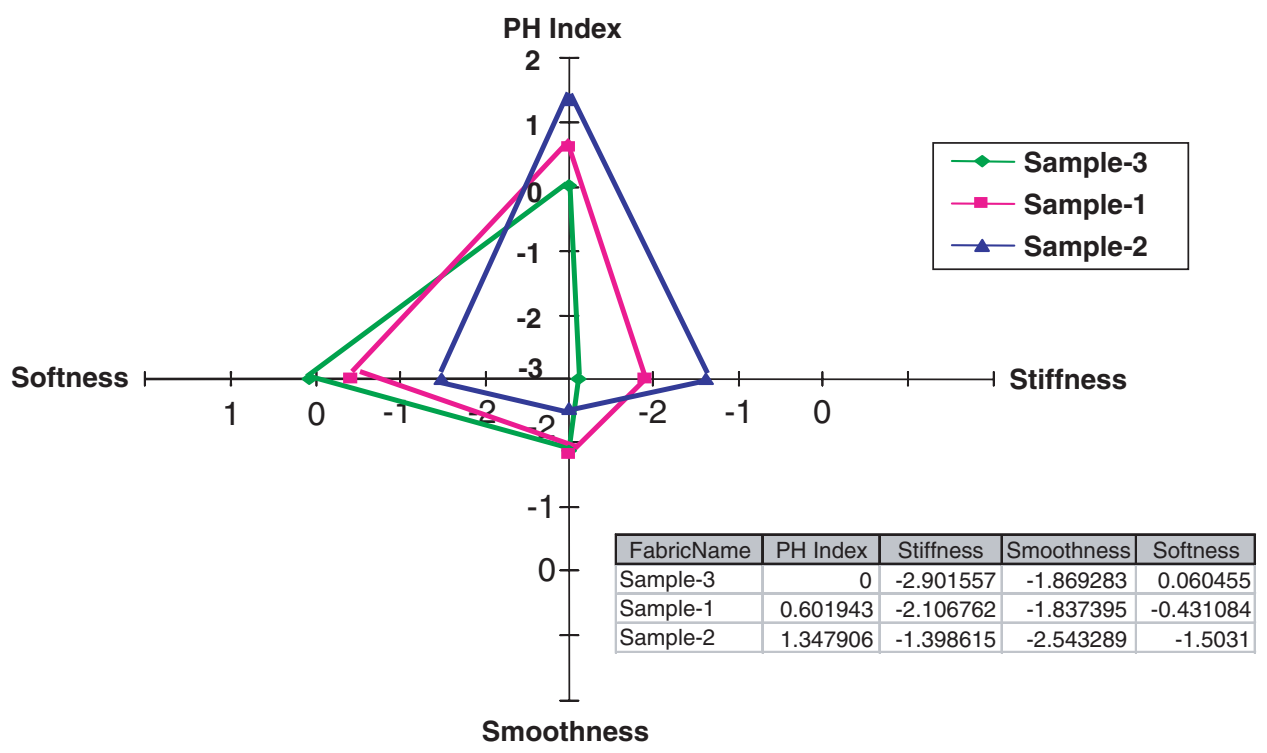

Figure 3: Fabric fingerprints. 
(a)

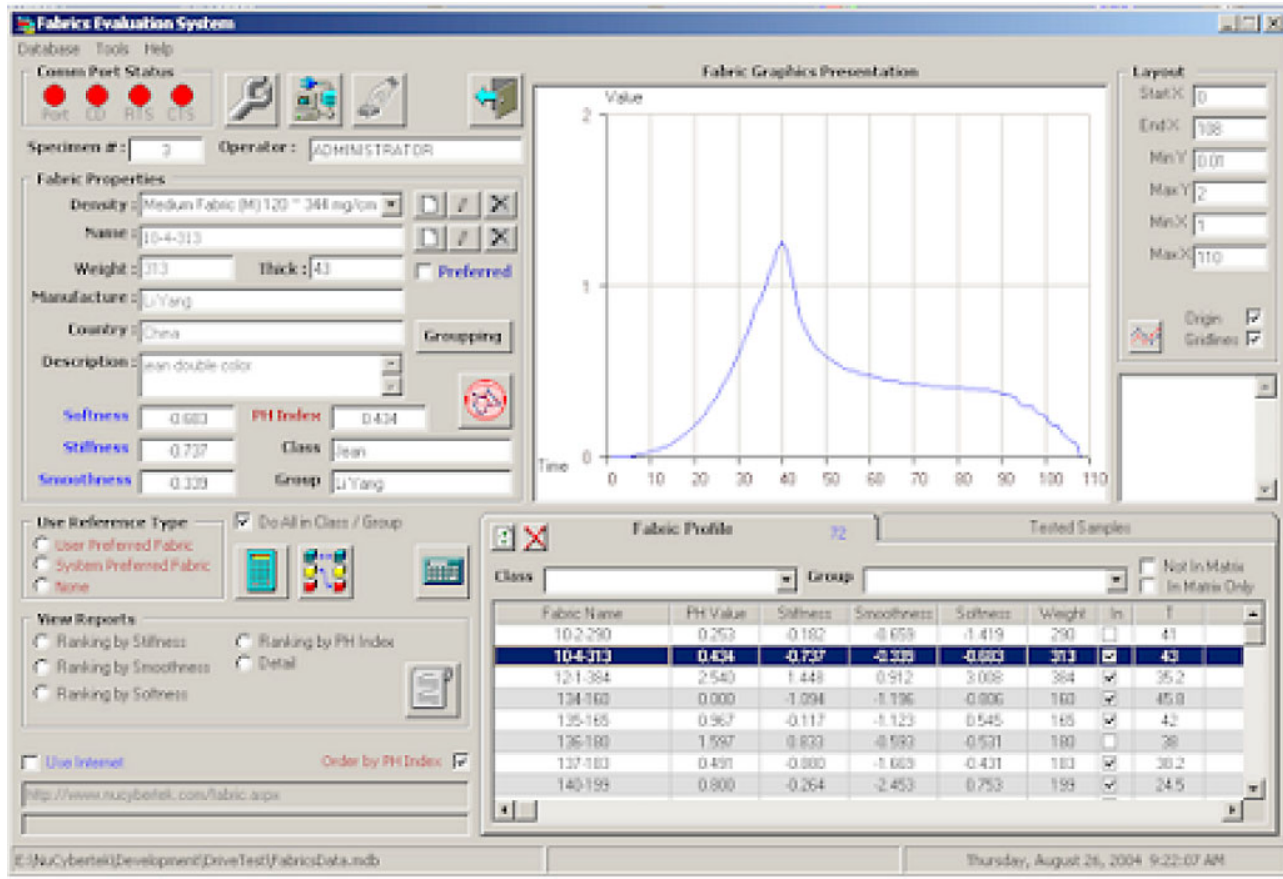

(b)

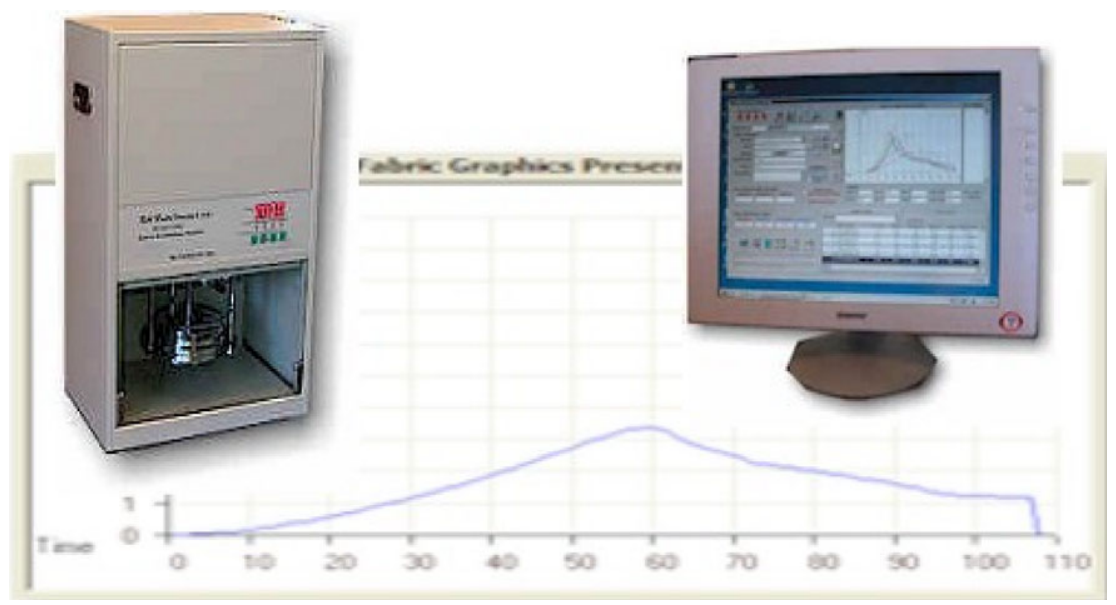

Figure 4: Photographs of the prototype: (a) system GUI; (b) instrument system.

Recently, we have built a prototype of the instrument based on the principles described above with a graphic user interface (GUI) and a system control, and data process software installed on a computer, as shown in Fig. 4.

\section{MORE APPLICATIONS}

This pattern recognition method and the instrument system have several other related applications including fabric drape and fabric wrinkle recovery evaluation; both these properties are human visual responses and are just as important as fabric hand. 
5.1 Fabric drape evaluation

Fabric drape refers to the fabric shape or profile when held at the edge, such as used for curtains, or the way a fabric covers an object when used as a tablecloth or a skirt; sometimes called the fabric formability in this case. Mechanically speaking, fabric drape is the fabric's response towards gravity due to its own weight. However, fabrics with identical mass can show very diverse drape behaviors, determined by the same mechanical properties defining fabric hand [41]. Therefore, it becomes obvious that fabric hand and drape are interconnected in a consistent way. Any difference in overall fabric hand, or in the individual hand attribute, can be used or interpreted as the difference in fabric drape behavior or fabric formability. The fabric extraction test is in fact a forced drape.

\subsection{Fabric wrinkle recovery evaluation}

Likewise, fabric wrinkle recovery is determined by the same group of mechanical properties as well [41]. During a fabric extraction test, fabric samples experience complex wrinkles yet with high repeatability. Thus, by testing a fabric twice using the present system, with a given recovery time interval between the tests, any differences in terms of the defined fabric hand parameters can be used as indicators of the ability of the fabric to recover from a given wrinkle.

\section{CONCLUSIONS}

Fabric tactile sense is widely viewed as an overall human response towards a fabric's mechanical properties and is therefore measurable by the test scheme introduced in this paper. The fabric extraction process deforms a fabric sample in a way similar to sensory evaluation, and all related information is thus contained in the extraction curve. The curve is then digitized into a data set $X$ via a data acquisition system in a computer for further pattern recognition analysis.

Next, the data set is treated by the K-L eigen transformation and results in the so-called feature set $Y$. The feature set $Y$ is much smaller in dimension than the original set $X$, and is orthogonal, i.e. all the components of $Y$ are independent of each other and they each contribute different yet complementary information in defining fabric hand. The relative importance of each component is also determined and the physical meanings of the first three most important components are ascertained for describing the different attributes of fabric hand, using some calibration techniques. For convenience, a weighted overall fabric hand $(\mathrm{PH})$ value is defined for fabric hand ranking, either in terms of consumer preference or in terms of a preassigned quality standard. The fingerprint technique introduced should be very useful for product comparison and analysis.

\section{REFERENCES}

[1] Basdogan, C., De, S., Kim, J., Muniyandi, M., Kim, H. \& Srinivasan, M.A., Haptics in minimally invasive surgical simulation and training. IEEE Computer Graphics and Applications, 24(2), pp. 56-64, 2004.

[2] DiMaio, S.P. \& Salcudean, S.E., Needle insertion modeling and simulation. IEEE Transactions on Robotics \& Automation, 19, pp. 864-875, 2003.

[3] Egawa, M., Oguri, M., Hirao, T., Takahashi, M. \& Miyakawa, M., The evaluation of skin friction using africtional feel analyzer. Skin Research and Technology, 8, pp. 41-51, 2002.

[4] Gepshtein, S. \& Banks, M.S., Viewing geometry determines how vision and haptics combine in size perception. Current Biology, 13, pp. 483-488, 2003.

[5] Griffiths, P. \& Kulke, T., Clothing movement—visual sensory evaluation and its correlation to fabric properties. Journal of Sensory Studies, 17, pp. 229-255, 2002. 
[6] Hillis, J.M., Ernst, M.O., Banks, M.S. \& Landy, M.S., Combining sensory information: mandatory fusion within, but not between, senses. Science, 298, pp. 1627-1630, 2002.

[7] Khatib, O., Brock, O., Chang, K.-S., Ruspini, D., Sentis, L. \& Viji, S. Human-centered robotics and interactive haptic simulation. International Journal of Robotics Research, 23, pp. 167-178, 2004.

[8] Oakley, I., Brewster, S. \& Gray, P., Communicating with feeling. Proc. of the 1st Int. Workshop on Haptic Human-Computer Interaction, eds. S. Brewster \& R. Murray-Smith, Springer-Verlag: Berlin, pp. 61-68, 2001.

[9] Rabin, E. \& Gordon, A.M., Influence of fingertip contact on illusory arm movements. Journal of Applied Physiology, 96, pp. 1555-1560, 2004.

[10] Richards, C., Rosen, J., Hannaford, B., Pellegrini, C. \& Sinanan, M., Skills evaluation in minimally invasive surgery using force/torque signatures. Surgical Endoscopy-Ultrasound and Interventional Techniques, 14, pp. 791-798, 2000.

[11] Stone, H. \& Sidel, J.L., Sensory Evaluation Practice. Academic Press, Inc.: San Diego, CA, 1993.

[12] Zimmermann, H.J., Fuzzy Set Theory and Its Applications, 4th edn, Kluwer: Boston, 2001.

[13] Dijksterhuis, G.B., Multivariate Data Analysis in Sensory and Consumer Science, Food \& Nutrition Press, Inc.: Trumbull, CT, 1997.

[14] Loden, M., Olsson, H., Skare, L. \& Axell, T., Instrumental and sensory evaluation of the frictional response of the skin following a single application of 5 moisturizing creams. Journal of the Society of Cosmetic Chemists, 43, pp. 13-20, 1992.

[15] Giboreau, A., Navarro, S., Faye, P. \& Dumortier, J., Sensory evaluation of automotive fabrics: the contribution of categorization tasks and nonverbal information to set-up a descriptive method of tactile properties. Food Quality and Preference, 12, pp. 311-322, 2001.

[16] Bishop, D.P., Fabrics: Sensory and Mechanical properties, Textile Progress, Vol. 26, No. 3, The Textile Institute: Manchester, UK, 2003.

[17] Dillon, P., Moody, W., Bartlett, R., Scully, P., Morgan, R. \& James, C., Sensing the fabric: to simulate sensation through sensory evaluation and in response to standard acceptable properties of specific materials when viewed as a digital image. Proc. of the 1st Int. Workshop on Haptic Human-Computer Interaction, eds. S. Brewster \& R. Murray-Smith, Springer-Verlag: Berlin, pp. 205-217, 2001.

[18] Binns, H., The discrimination of wool fabrics by the sense of touch. British Journal of Psychiatry, 16, pp. 237-247, 1926.

[19] Peirce, F.T., The 'handle' of cloth as a measurable quantity. Journal of the Textile Institute, 21, pp. T377-416, 1930.

[20] Brand, R.H., Measurement of fabric aesthetics: analysis of aesthetic components. Textile Research Journal, 34, p. 791, 1964.

[21] Vaughn, E.A. \& Kim, C.J., Study on Fabric Hand, Parts I, II, and III, Technical Conference, American Association of Textile Chemists and Colorists, 1975.

[22] Elks, B.C. \& Carnsworrthy, R.K., A review of techniques for the assessment of hand. Textile Research Journal, 50, p. 231, 1980.

[23] Kawabata, S., Examination of effect of basic mechanical properties of fabrics on fabric hand. Mechanics of Flexible Fiber Assemblies, NATO Advanced Study Institute Series, Sijthoff \& Noordhoff: Germantown, MD, pp. 405-417, 1980.

[24] Kawabata, S., The Standardization and Analysis of Handle Evaluation, 2nd edn, The Textile Machinery Society of Japan: Osaka, Japan, 1980. 
[25] Pan, N., Yen, K.C., Zhao, S.J. \& Yang, S.R., The objective measure for fabric total handle. Textile Research Journal, 58, p. 438, 1988.

[26] Pan, N., Yen, K.C., Zhao, S.J. \& Yang, S.R., The objective measures for fabric primary handle. Textile Research Journal, 58, p. 531, 1988.

[27] Pan, N., Yen, K.C., Zhao, S.J. \& Yang, S.R., Fuzzy cluster analysis for fabric handle sorting. Textile Research Journal, 58, p. 565, 1988.

[28] Tester, D. \& De Boos, A., Get it right FAST time. Textile Horizons, 10(8), p. 13, 1990.

[29] Mahar, T.J. \& Postle, R., International fabric handle survey. Proc. Objective Evaluation of Apparel Fabrics, eds. R. Postle, S. Kawabata \& M. Niwa, The Textile Machinery Society of Japan: Osaka, Japan, pp. 261-271, 1983.

[30] Mahar, T.J., Postle, R., Dhingra, R.C., Gupta, V.B., Kawabata, S., Niwa, M. \& Carnaby, G.A., The objective specification of the handle of men's suiting materials-a comparison of fabric handle assessments in India, Australia, Japan and New Zealand. Indian Textile Research Journal, 8, pp. 9-15, 1983.

[31] Behery, H.M., Comparison of fabric hand assessment in the United States and Japan. Textile Research Journal, 56, pp. 227-240, 1986.

[32] Postle, R., Fabric objective measurement technology: 1. Historical and background development. Textiles Asia, 20(7), pp. 64-66, 1989.

[33] Postle, R., Mahar, T.J. \& Dhingra, R.C., Measuring and interpreting of low stress fabric mechanical and surface properties. Part I: Precision of measurement. Textile Research Journal, 57, pp. 357-368, 1987.

[34] Alley, V.L., Jr. \& McHatton, A.D., A Proposed Quantitative Measure of Fabric Handle and the Relative Characterization of Some Aerospace Flexible Materials by Handle Moduli, AFGIrTR-76-0306, Special Report Number 200, 1976.

[35] Alley, V.L., Jr., Revised theory for the quantitative analysis of fabric hand. Journal of Engineering and Industry, 102, pp. 25-31, 1980.

[36] Pan, N. \& Yen, K.C., Pattern recognition method for fabric handle evaluation. Journal of China Textile Engineering Association, 5, pp. 731-734, 1984.

[37] Pan, N. \& Yen, K.C., Physical explanations of fabric extracting curve for fabric handle evaluation. Textile Research Journal, 62, pp. 279-290, 1992.

[38] Fukunaga, K., Introduction to Statistical Pattern Recognition, 2nd edn, Academic Press, Inc.: San Diego, CA, 1990.

[39] Kendall, M., Multivariate Analysis, Charles Griffin and Company Ltd: London, p. 170, 1975.

[40] Pan, N., Zeronian, H. \& Ryu, H.S., An alternative approach to the objective measurement of fabrics. Textile Research Journal, 63, pp. 33-43, 1993.

[41] Hearle, J.W.S., Grosberg, P. \& Backer, S., Structural Mechanics of Yarns and Fabrics, Vol. 1, Wiley-Interscience: New York, 1969. 